Revue d'histoire de l'Amérique française

RES REVUE D.HISTOIRE DE L'AMÉRIQUE FRANÇAISE

\title{
Les Canadiens veulent conserver le régime seigneurial (suite)
}

\section{Marcel-Joseph}

Volume 7, numéro 3, décembre 1953

URI : https://id.erudit.org/iderudit/301607ar

DOI : https://doi.org/10.7202/301607ar

Aller au sommaire du numéro

Éditeur(s)

Institut d'histoire de l'Amérique française

ISSN

0035-2357 (imprimé)

1492-1383 (numérique)

Découvrir la revue

Citer cet article

Marcel-Joseph (1953). Les Canadiens veulent conserver le régime seigneurial (suite). Revue d'histoire de l'Amérique française, 7(3), 356-391.

https://doi.org/10.7202/301607ar d'utilisation que vous pouvez consulter en ligne.

https://apropos.erudit.org/fr/usagers/politique-dutilisation/ 


\title{
LES CANADIENS VEULENT CONSERVER LE REGIME SEIGNEURIAL *
}

\author{
(suite)
}

\author{
CHAPITRE III \\ |MANQUE D'ESPACE |VITAL
}

Dalhousie et les immigrants - Les immigrants et les terres de la couronne - Les terres incultes des seigneuries - Le Comité des Terres de la Couronne - L'enquête - Session de 1820-21 - Session de 1822 - Session de 1823-24 - Plus de terres disponibles - Terres disponibles, conditions normales - Terres disponibles, mais obstacles naturels.

Le 18 juin 1810, le Comte de Dalhousie assumait les fonctions de gouverneur général du Canada ${ }^{1}$. Tout au long de l'été, il put assister au débarquement de milliers de ses compatriotes qui venaient recommencer leur vie en terre d'Amérique. D'une semaine à l'autre, il en arrivait sans cesse: 11,239 débarquèrent sur nos bords durant le premier été que Dalhousie passa à Québec². Mais à peine avaient-ils mis pied à terre, qu'ils se rembarquaient pour quelques autres semaines de navigation vers le haut du Fleuve. Entre le BasCanada français et le Haut-Canada anglais, ils n'hésitaient pas à choisir le second ${ }^{3}$.

Très peu restèrent "dans cette Province, moins par inclination que par accident, maladie ou manque de moyens pécuniaires pour

* Voir RHAF, vol. VII, no 1: 45-63; no 2: 224-240.

1. J. Bouchette, The British Dominions in North America; or a Topographical and Statistical Description of the Provinces of Lower and Upper Canada. New Brunswick, Nova Scotia, the Islands of Newfoundland, Prince Edward, and Cape Breton. Including Considerations on Land-Granting and Emigration. To which Are Annexed. Statistical Tables and Tables of Distances, etc. (2 vol., London, 1831), 1: 448.

2. A. Stuart, "Quatrième rapport", 6 mars 1821, dans Premier rapport, 2: 38.

3. Ibid., 41. 
pouvoir aller dans l'intérieur"4; ou encore, parce que tel ou tel Anglais, seigneur ou agent, les attirèrent sur leurs terres. Edward Hale en accueillit dans sa seigneurie de Portneuf ${ }^{5}$. Roderick Mc Kenzie établit de soixante à quatre-vingts familles écossaises dans sa seigneurie de Laplaine, en arrière de Terrebonne $e^{6}$. John Macnider, seigneur de Mitis, décida quarante familles d'immigrants à aller se fixer dans le Bas-du-Fleuve: chaque famille y reçut une très grande terre de cent quarante à deux cents arpents carrés, chargée d'une rente en argent de douze schellings et demi, dont elle était exempte pendant les deux premières années; chaque famille reçut en outre, de la part du seigneur, des avances en argent pendant les deux premières années ${ }^{7}$. Shadgett, simple particulier, fit aussi cuvre louable au point de vue britannique; il raconte son histoire de la façon suivante: "J'ai moi-même demandé à l'Honorable A.-L. J.-Duchesnay, au printemps de 1821, "qu'il me fát permis en premier lieu de former un Village Anglois près du Lac de Beauport, maintenant Lac Duchesnay, et ensuite d'établir des Emigrés Anglois sur les parties non concédées de la Seigneurie"; et ma proposition a été reçue. Les premiers travaux ont été entrepris au cours de l'hiver de 1821. Nous comptons actuellement (1823) cent soixante-huit concessionnaires dont soixante à quatre-vingts sont déjà établis. Ce sont tous des gens d'une classe supérieure, savoir: des marchands, des gens de profession, des gens employés aux travaux du roi et des cultivateurs; "les Emigrés n'ayant plus aucune répugnance pour la tenure seigneuriale, car ils voient qu'ils peuvent devenir possesseurs des terres sans achat, et qu'ils ont ainsi leur argent comptant à eux pour les défricher et cultiver"." Voilà pour l'initiative privée.

4. Ibid., 38.

5. Témoignage d'Edward Hale, Neilson et Cowan, éd., Journaux de la Chambre d'Assemblee du Bas-Canada, 32 (1823), Appendice T.

6. Letters from the Curates of the respective Parishes of Lower-Canada, referred to in the Fifth Report of the Special Committee on the Waste Lands of the Crown (a l'avenir: Letters from the Curates ] ([Québec ], 1823), 61 et 66. L'un dit des Ecossais, l'autre, des Américains.

7. Témoignage de John Macnider, Neilson et Cowan, éd., Journaux de la Chambre d'Assemblée du Bas-Canada, 32 (1823), Appendice T; J. Neilson, éd., Journaux de la Chambre d'Assemblee du Bas-Canada, 17 (1808): 347.

8. Témoignage de Shadgett, Neilson et Cowan, éd. Journaux de la Chambre d'Assemblé du Bas-Canada, 32 (1823), Appendice T. 
Mais "des trente-neuf mille cent soixante et trois Émigrés, qui sont arrivés au port de Québec durant les quatre dernières années, [ 1817-18-19-20] pas plus de cent familles n'ont trouvé un refuge et la subsistance sur les terres incultes et non-concédées de la Couronne." Il est honteux, pensa Dalhousie, qu'une colonie de l'Empire ne soit pas plus accueillante aux Britanniques. Que n'a-t-on établi ces gens sur les immenses terres incultes que le roi possède en ce pays?

A l'ouverture de la session, le 16 décembre 1820, Dalhousie demanda de l'aide pour ses compatriotes que la vague de l'infortune jetait sur nos bords, tout en faisant de la question une affaire d'exploitation des terres de la couronne et en insistant sur les avantages que la Province retirerait de la mise en valeur de ces terres: Un pays vaut par ses hommes; l'acquisition de ces milliers de sujets britanniques, serait tout au profit du Canada. Néanmoins, lors même qu'on ne réussirait pas à les retenir sur les terres de la couronne, il faudrait quand même faciliter l'exploitation de ces dernières, car "le Bas-Canada possède une population suffisamment ample pour établir ces Terres incultes et aussi celles encore non-concédées dans les Seigneuries"10. S'étant fait un devoir, comme il le dit lui-même, de visiter, au cours de l'été, autant de parties du pays que les circonstances le lui avaient permis, il était déjà au fait du grand malaise dont souffrait la Province à cette époque. Il conviendrait, continuait-il, d'ériger des églises et d'ouvrir des chemins pour encourager les Canadiens "à étendre leurs établissements davantage"."

C'est pour répondre à cet appel du gouverneur qu'un comité de neuf membres fut créé, au cours de janvier: le Comité des Terres de la Couronne. Il se composait des députés J.-T. Taschereau, Jean Bélanger, Louis Lagueux, Frs Fournier, Jacob Oldham, F. Blanchet, Andrew Stuart, John Neilson et D.-B. Viger ${ }^{12}$.

9. A. Stuart, "Quatrième rapport", 6 mars 1821, dans Premier rapport, 2: 39.

10. J. Neilson, ed., Journaux de la Chambre d'Assemblee du Bas-Canada, 30 (1821): 33 .

11. Ibid.

12. Ibid., 79; J. Bouchette, The British Dominions in North America; or a Topographical and Statistical Description of the Provinces of Lower and Upper Canada, New Brunswick, Nova Scotia, the Islands of Newfoundland, Prince Edward, and Cape Breton, 1: 431 . 
L'établissement des immigrants "est arrêté dans son cours faute d'aide de la part de la Législature"13 a a vait dit le gouverneur. Là n'est pas ła cause, répondit le Comité. Ce qui a éloigné les immigrants c'est, d'une part, la façon dont les Townships sont divisés et, d'autre part, la conduite mesquine des employés du bureau des terres de la couronne et des membres de la Commission des Biens des Jésuites ${ }^{14}$.

Laissant les émigrés à leur sort, le comité s'occupa surtout de ce qui avait trait aux Canadiens, aux terres de la couronne et aux seigneuries. Dans le but d'obtenir des renseignements de première main et de tirer les choses au clair, un questionnaire détaillé fut adressé aux propriétaires de seigneurie et aux curés de paroisse de la Province ${ }^{15}$. Après quoi on discuta de tenures. Quiconque a lu Munro' ${ }^{16}$, préjuge qu'on y dénigra la tenure seigneuriale au profit de la tenure libératrice importée d'Angleterre. Bien au contraire. Dès son premier rapport, le comité prit une position bien tranchée et se prononça carrément pour la tenure seigneuriale: "Votre Comité est d'opinion qu'une des causes principales qui ont retardé l'établissement des Terres incultes de la Couronne dans le Bas-Canada, a été l'abandon de l'ancienne manière de concéder les Terres en Fiefs ou en Seigneuries, et l'introduction de la Tenure en franc et commun Soccage, Tenure nouvelle et inconnue au peuple de ce Pays ${ }^{17}$." Cette appréciation était de leur cru et non pas suggérée par les réponses des curés: ils n'en avaient encore reçu aucune; ni par celles des seigneurs: ils ne répondirent jamais ${ }^{18}$.

Voilà un témoignage, voici des explications: "Votre Comité en procédant à l'examen des avantages et désavantages comparatifs de l'ancienne Tenure de ce Pays et de la Tenure en Franc et Commun Soccage, est venu à une conclusion que la première possède sur la (1821): 33.

13. J. Neilson, éd., Journaux de la Chambre d'Assemblée du Bas-Canada, 30

14. A. Stuart, "Quatrième rapport", 6 mars 1821, dans Premier rapport, 2: 38.

15. A. Stuart, "Premier rapport", 12 février 1821, dans Premier rapport, 1: 3.

16. W. B. Munro, "The Seigneurial System and the Colony", Canada and its Provinces $a$ History of the Canadian People and their Institutions by one Hundred Associates (23 vol., Toronto, 1914-1917), 2: 587.

17. A. Stuart, "Premier rapport", 12 février 1821, dans Premier rapport, 1: 4. 18. Ibid., 3. 
dernière de grands avantages dans l'établissement d'un Pays nouveau. Votre Comité n'a pas jugé nécessaire de s'enquérir sur les avantages comparatifs de ces Tenures dans un état plus avancé de la Société, laissant à la sagesse de la postérité à pourvoir des remèdes à ces inconvénients lorsqu'ils auront lieu ${ }^{19}$." C'était l'option pour la terre. De commerce, d'industrie, personne ne parle; on n'y pense même pas. La postérité y pourvoira. Pour l'instant, vive l'agriculture qui apporte régulièrement le pain quotidien. Cela suffit. Vive aussi la tenure seigneuriale qui donne au plus pauvre une terre de laquelle il puisse tirer sa subsistance. Le seul inconvénient du système seigneurial, déclare le Comité, consistait dans la juridiction civile et quelquefois criminelle attachée aux seigneuries. Les nouveaux maîtres du pays, en abolissant ce pouvoir judiciaire, ont supprimé le seul sujet de plainte des Canadiens contre le système ${ }^{20}$. (N'en déplaise à T. Guerin qui soutient l'opinion contraire) ${ }^{21}$. Par ailleurs, les avantages du système français sont multiples. Le premier, c'est qu'il ne coûte pas cher. Les gens qui sont obligés de prendre une terre non défrichée, sont pauvres, sans cela ils se tireraient d'affaire autrement. Le meilleur système pour eux, est le moins onéreux, c'esta-dire le système français des seigneuries. "Sous cette Tenure celui qui faisoit un Établissement avoit plusieurs avantages: il n'étoit pas obligé de débourser aucun argent pour sa Terre. Quelque petit fonds qu'il possédât il le pouvoit entièrement employer à l'amélioration de sa Terre ${ }^{22}$." Quant au seigneur, il était obligé, en certains cas, de faire des chemins. De plus, "Il devoit bâtir un Moulin, et la Mouture étoit payée à un taux plus bas (1-14) qu'il ne l'est maintenant [ 1821 ] dans les États-Unis où il y a la plus grande concurrence (1-12)." Comme le seigneur "étoit intéressé à effectuer des Établissemens pour garantir sa Seigneurie de confiscation", il accordait protection, appui, assistance au nouveau censitaire, lequel "avoit tout cela pour une modique Rente annuelle". Autre avantage, le système des seigneuries a produit en ce pays une division égale des

19. Ibid., 8.

20. Ibid., 9.

21. T. Guerin, Feudal Canada. The Story of the Seigniories of New France (Montréal, 1926), 139.

22. A. Stuart, "Premier rapport", 12 février 1821, dans Premier rapport, 1: 8-9. 
terres, "chose favorable au bonheur des hommes". Aussi le Comité recommandait-il l'érection d'un nombre suffisant de seigneuries pour assurer des terres au "surplus de la population du pays"23.

Surplus... on était donc à l'étroit quelque part, car, où il y a surplus, il y a manque. Manquait-on d'espace vital, de terre? Certains en doutaient. Pour John Neilson, c'était là un préjuge ${ }^{24}$. Poursuivant ses investigations, le Comité en vint à la conclusion qu'il restait encore, dans les seigneuries déjà existantes, de très grandes étendues de terre propres à la culture, qui n'étaient pas encore concédées. Pourquoi ne l'étaient-elles pas, puisqu'il y avait surplus de population? "Dans l'opinion de votre Comité, le principal obstacle a l'Établissement des Seigneuries non-concédées de cette Province, a été la négligence de l'Administration Coloniale à mettre en force les Lois du pays concernant les Concessions en Censive des différentes Seigneuries de cette Province". Sous le gouvernement de Sa Majesté Très-Chrétienne on pouvait se plaindre des exactions des fonctionnaires, et des services militaires onéreux, mais on "avoit tout lieu d'être satisfait de la sagesse et de l'attention que mettoit Sa Majesté dans cette branche de l'Administration. Depuis la Conquête l'Administration Coloniale a souffert que ces Lois demeurassent sans execution", voila pourquoi la Province compte, à l'heure actuelle, à la fois des terres sans hommes et des hommes sans terre ${ }^{25}$.

La session de 1820 - 21 tirant à sa fin, les réponses des curés de paroisse, au questionnaire adressé par le Comité, furent réservées pour la session suivante. Lorsque celle-ci s'ouvrit, on remit sur pied le Comité des Terres de la Couronne, lequel reprit ses séances le 5 janvier 1822. Il était constitué des sept députés qui en avaient fait partie l'année précédente: Andrew Stuart,|John Neilson, Frs Fournier, D.-B. Viger, F. Blanchet, Jacob Oldham, Louis Lagueux ${ }^{26}$. Il éplucha

23. Ibid., 10.

24. "Plan par John Neilson, Écuyer, pour former un nouvel Établissement étendu sur le côté nord du Fleuve Saint-Laurent, dans les environs de Québec", octobre 1819, Appendice du XXXIIIe volume des Journaux de la Chambre d'Assemblée de la province du Bas-Canada, Appendice R. Appendice A.

25. A. Stuart, "Troisième rapport", dans Premier rapport, $2: 1$.

26. J. Neilson, éd., Journaux de la Chambre d'Assemblée du Bas-Canada, 31 (1822): 54 ; J. Bouchette, The British Dominions in North America; or a Topographical and Statistical Description of the Provinces of Lower and Upper Canada, New Brunswoick, Nova Scotia, the Islands of Newfoundland, Prince Edward, and Cape Breton, 1: 431 . 
les lettres des curés de paroisse du Bas-Canada ${ }^{27}$, fit la lumière sur bien des points, mais ne put, encore cette fois, terminer son enquête.

Un nouvel ajournement et une nouvelle reprise à la session de 1823-24, s'avérèrent nécessaires ${ }^{28}$. Cette fois, les députés Andrew Stuart, Frs Saint-Onge, Joseph Perrault, Michael O'Sullivan, D.-B. Viger, Hugues Heney, Ls. Bourdages et Pierre Bureau furent désignés pour poursuivre l'enquête et autorisés à envoyer quérir personnes et documents jugés nécessaires ${ }^{29}$. Les dépositions des témoins cités à la barre, confirmèrent et complétèrent les dires des curés, étudiés l'année précédente.

L'enquête démontra que l'expression "plus de terres" entendue partout, avait des acceptions différentes selon les lieux. Cependant, appliquée aux paroisses et aux seigneuries suivantes, elle devait être prise au pied de la lettre:

DISTRICT DE QUEBEC

Saint-Ambroise ${ }^{30}$,

Neuville,

Les Ecureuils,

Seigneurie Saint-Augustin ou Demaure ${ }^{31}$,

Sainte-Famille, Isle d'Orléans,

Saint-François, Isle d'Orléans ${ }^{32}$,

Saint-Jean, Isle d'Orléans ${ }^{33}$,

Saint-Laurent, Isle d'Orléans,

Saint-Pierre, Isle d'Orléans ${ }^{34}$,

Rivière-Ouelle ${ }^{35}$,

Sainte-Anne de la Pocatière ${ }^{36}$,
Saint-Roch des Aulnets ${ }^{37}$,

Saint-Jean Port Joli ${ }^{38}$,

L'Islette ${ }^{39}$,

Saint-Valier,

Belle-Chasse ${ }^{40}$,

Saint-Michel ${ }^{41}$,

Beaumont, trois seigneuries: Beaumont, Vincennes, Livaudière ${ }^{42}$,

Saint-Charles de Bellechasse, trois seigneuries: Beaumont, Livaudière, Lamartinière ${ }^{43}$,

Saint-Joseph de la Pointe-Lévit4, Seigneurie de Sillery ${ }^{45}$,

27. Letters from the Curates.

28. A. Stuart, "Rapport", 18 février 1822, J. Neilson, éd., Journaux de la Chambre d'Assemblee du Bas-Canada, 31 (1822), Appendice N.

29. Appendice du XXXIIIe volume des Journaux de la Chambre d'Assemblée de la province du Bas-Canada, Appendice R; J. Bouchette, The British Dominions in North America, 1: 431.

30. Letters from the Curates, 10.

31. Ibid., 11.

32. Ibid., 15.

33. Ibid., 16.

34. Ibid., 17.

35. Ibid., 20.

36. Ibid., 23.

37. Ibid., 26.

38. Ibid., 28.

39. Ibid., 30.

40. Ibid., 31.

41. Ibid., 33.

42. Ibid., 35.

43. Ibid., 37.

45. Témoignage de Michel Berthelot, 9 décembre 1823, Appendice XXXIIIe volume des Journaux de la Chambre d'Assemblée de la province du Bas-Canada, Appendice $R$, Appendice A. 


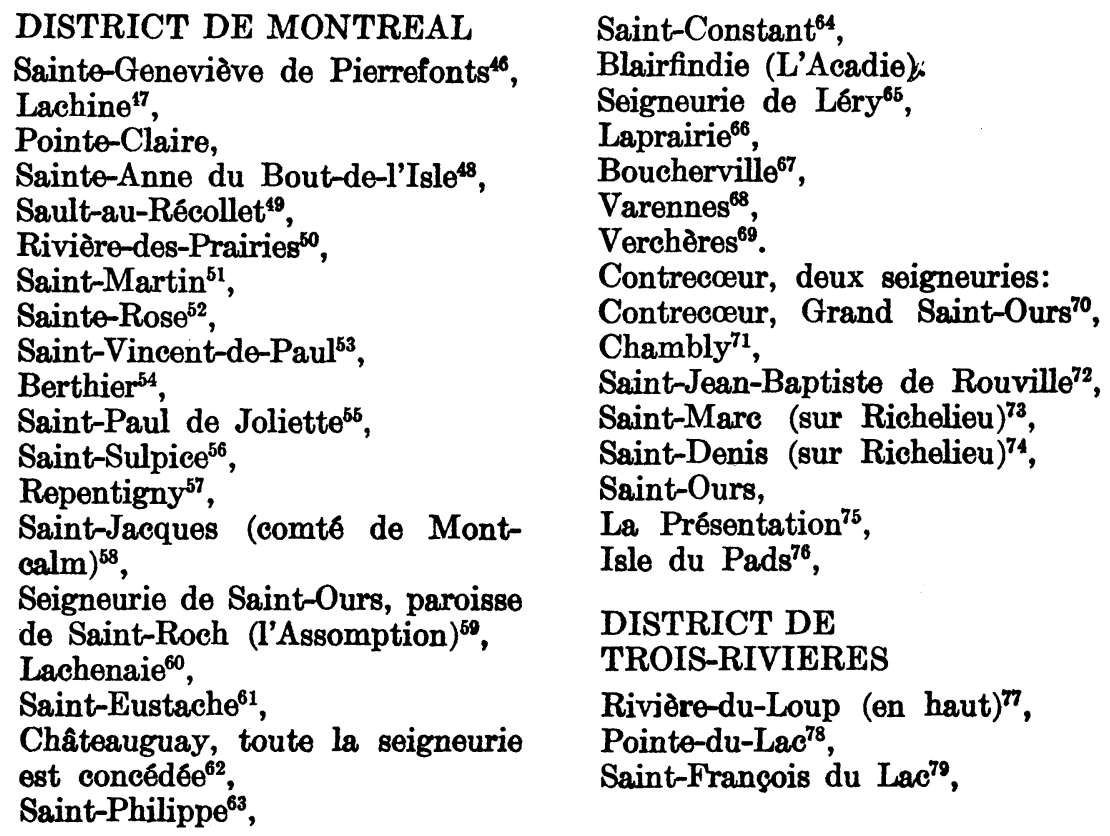

Dans la paroisse de Saint-Ours, on n'avait pas encore commencé à en souffrir cependant: "Jusqu'd ce jour tous ceux qui ont demandé des terres les ont eues, au taux ci-dessus" (1 sol par arpent et 1/2 minot de blé par 20 arpents). Mais désormais c'est fini, il n'en reste

46. Letters from the Curates, 46.

63. Ibid., 86.

47. Ibid., 47.

48. Ibid., 48 .

49. Ibid., 49.

50. Ibid., 50.

51. Ibid., 51 .

52. Ibid., 52.

53. Ibid., 53.

54. Ibid., 54.

55. Ibid., 56.

56. Ibid., 57.

57. Ibid., 58.

58. Ibid., 59 .

59. Ibid., 60.

60. Ibid., 63.

61. Ibid., 71 .

64. Ibid., 88.

65. Ibid., 91.

66. Ibid., 92.

67. Ibid., 93.

68. Ibid., 95.

69. Ibid., 96.

70. Ibid., 97.

71. Ibid., 98.

72. Ibid., 99.

73. Ibid., 101.

74. Ibid., 102.

75. Ibid., 104.

76. Ibid., 106.

77. Ibid., 110.

62. Ibid., 84 .

78. Ibid., 111.

79. Ibid., 115. 
aucune $^{80}$. Toutes les autres paroisses étaient dans des situations analogues à celles des trois suivantes: Saint-Jean Port-Joli: "Un grand nombre de personnes ont les moyens, et même désirent s'établir dans cette Paroisse, où elles sont nées, et où demeurent tous leurs parents; mais elles ne peuvent le faire, parce que toutes les terres sont prises ${ }^{81}$." Saint-Jacques (comté de Montcalm): il ne reste ici que neuf arpents de sol impropre à la culture. Six cents jeunes hommes, à part cent familles sans terre, en voudraient. Mais les Townships voisins sont déjà pleins d'étrangers. Nos jeunes hommes "se ruinent par des voyages entrepris en vue de se procurer les moyens de s'établir dans cette Paroisse, ce qui est une lourde dépense..."; mais c'est peine perdue, car "cette robustesse qui est si nécessaire au cultivateur est gaspillée dans des contrées lointaines par ces jeunes vagabonds; leur Religion aussi décroît, et à leur retour à la maison ils augmentent simplement le nombre de ces étrangers vauriens qui abondent constamment dans cette région" 82 . Saint-Paul de Joliette: la paroisse compte 2,200 âmes. Le nombre des familles propriétaires, est de 300. Un cinquième des paroissiens sont dans la mendicité: leurs terres sablonneuses ne produisant rien. Aucune terre n'est disponible. La paroisse contient un excédent de population de 250 cultivateurs non mariés, âgés de plus de vingt et un ans, et de 360 filles non mariées, âgées de plus de dix-huit $a^{8} s^{83}$.

Ajoutons à cette liste, les endroits où des étendues de terre plus ou moins considérables n'étaient pas concédées et ne pouvaient l'être parce que le sol défiait toute culture:

L'immense seigneurie de Beaupré, en aval de Québec sur la rive nord: il reste ici une étendue considérable de terre; malheureusement, elle est impropre à la culture. De plus, "la rigueur du climat est telle, que le blé gèle presque chaque année". Aussi les jeunes émigrent-ils vers les districts de Trois-Rivières et de Montréal, constatent les curés de

Sainte-Anne de Beaupré $e^{84}$, Saint-Joachim ${ }^{85}$,
L'Ange-Gardien ${ }^{86}$, Château-Richer ${ }^{87}$.
80. Ibid., 104.
81. Ibid., 29.
82. Ibid., 59.
83. Ibid., 56.
84. Ibid., 4.

85. Ibid., 5 .

86. Ibid., 2.

87. Ibid., 1. 
Les seigneuries de Livaudière et de La Durantaye, sur la rive sud: le peu de terre dont les propriétaires disposaient encore en 1821, n'était pas arpenté, dépourvu de route pour s'y rendre, et à peine cultivable: sa principale production étant le caillou ${ }^{88}$.

Le curé de Rivière-Ouelle avait la note juste, lorsqu'il écrivait: dans le district de Québec, ce qui retarde la colonisation agricole dans les vieilles seigneuries, c'est la proximité des montagnes au nord et au sud du Saint-Laurent. Les terres au delà de ces montagnes, offrent un sol aride et rocailleux, coupé d'autres montagnes. On n'y trouve que peu de terre favorable à la culture ${ }^{89}$.

Dans le district de Montréal, la paroisse de Terrebonne, qui occupait à peine un tiers de la surface de la seigneurie, portait mal le nom de cette dernière: les terres qui ne sont pas prises, sont de texture sablonneuse et ne peuvent pas faire vivre un cultivateur ${ }^{90}$.

De ces endroits, aussi bien dire qu'il ne restait pas de terre.

Est-ce à dire qu'il n'y en avait plus nulle part ? D'après l'augmentation de la population, calculait Joseph Bouchette, il devrait y avoir la moitié de la terre seigneuriale de défrichée à l'heure actuelle; mais le défrichement $n$ 'est pas si avancé que cela ${ }^{91}$. De fait, quelques curés avouent qu'il reste des terres non concédées dans leur paroisse, qu'elles sont propres à la culture et que les gens en obtiennent facilement.

Dans le district de Québec, à Sainte-Marie de Nouvelle-Beauce, par exemple. Il y restait beaucoup de bonnes terres disponibles; elles étaient arpentées et reliées à la paroisse par des chemins. Depuis quelque temps, lit-on dans le rapport de cette paroisse, presque tous les jeunes hommes ont pris des terres ${ }^{92}$.

Dans le Bas-du-Fleuve, les paroisses de Rivière-du-Loup (974 âmes), Kakouna (849 âmes) et l'Ile Verte (732 âmes) contenaient beaucoup de terre non défrichée, comme l'indique d'ailleurs le chiffre

88. Ibid., 39.

89. Ibid., 22.

90. Ibid., 64 .

91. Témoignage de Joseph Bouchette, 30 décembre 1823, Appendice du $X X X I I I$ e volume des Journaux de la Chambre d'Assemblee de la province du BasCanada, Appendice R, Appendice A.

92. Letters from the Curates, 41. 
de leur population. L'Ile-Verte, toutefois, faisait figure de paroisse fermée: "la plupart des tenanciers tiennent leur terre en fief:c'est-àdire:- qu'ils possèdent une ferme de deux ou trois arpens, et même plus, de front, par deux lieues de profondeur, de sorte que le seigneur ne peut faire aucune nouvelle concession ${ }^{93}$. Pour ce qui est de Ka kouna, de Rivière-du-Loup, et des autres propriétés d'Alexander Fraser,... je puis affirmer que les jeunes hommes sont avides d'obtenir des Concessions dans ces lieux, et je n'ai aucun doute que dans deux ou trois ans au plus, la Seigneurie d'Alexander Fraser... sera toute concédée, et en partie établie ${ }^{94}$." On y accourait, en effet, de RivièreOuelle: "Un grand nombre de pères de famille de cette paroisse, qui étaient capables de fournir à leurs enfants des bestiaux et des provisions, sont allés pour eux à vingt ou trente lieues d'ici, à Rivièredu-Loup, Kakouna, Trois-Pistoles, et même aussi loin que Rimouski. Au cours des vingt dernières années, beaucoup se sont établis là, sur des terres propres à la culture ${ }^{95}$." On y venait même de Saint-Roch des Aulnets: Chaque année, nous en voyons quelques-uns aller défricher des terres dans des seigneuries distantes de vingt ou trente lieues et même davantage ${ }^{96}$.

Plus on descendait le Fleuve, plus la population était clairsemée: "La Seigneurie de $\mathrm{Ha} \mathrm{Ha}$, qui est entre les Trois-Pistoles et le Bic, est peu établie; le sol paroit bon entre les Montagnes... Le Bic est très peu établi... La Seigneurie de la Rivière Oty est peu établie, montagneuse et d'un sol sablonneux et léger." Rimouski compte une centaine de familles. Les chemins sont beaux jusqu'à l'Ansedu-Coq, ensuite ils sont rudes jusqu'à Mitis; le sol est excellent. De l'Anse-du-Coq à Mitis, distance de deux lieues, il y a peu d'établissements; les trois autres lieues sont concédées jusqu'à la rivière du Grand Mitis. Le témoin qui donnait ces renseignements aux membres du Comité, était précisément le seigneur de Mitis, John Macnider, grâce aux largesses duquel le premier rang de cette sei-

93. Bouchette ne nomme qu'un fief, Pachot, dans le comté de Rimouski: J. Bouchette, The British Dominions in North America; or a Topographical and Statistical Description of the Provinces of Lower and Upper Canada, New Brunswick, Nova Scotia, the Islands of Newfoundland, Prince Edward, and Cape Breton, 1: 179.

94. Letters from the Curates, 18.

95. Ibid., 22.

96. Ibid., 27. 
gneurie était occupé par quarante familles d'immigrants. Après Mitis, c'était la forêt jusqu'à Matane. Si éloignée que fût cette seigneurie, elle n'était tout de même pas déserte, une trentaine de familles y étaient implantées ${ }^{97}$.

Dans le district de Trois-Rivières, une paroisse nous est connue, Gentilly, où il restait des terres non concédées et où les gens en obtenaient facilement, parce que le seigneur de l'endroit respectait encore, en 1820, les us et coutumes établis sous le gouvernement français: "La Seigneurie ou Paroisse de Gentilly a deux lieues de profondeur. Quatre Concessions sont établies", la ligne de front du quatrième rang a été tirée l'an dernier, et déjà presque toutes les terres sont concédées, parce que le seigneur demande une rente modique. "Il reste à concéder... environ deux rangs de 30 arpens de profondeur". Il n'y a pas de route sur les terres non concédées, et elles ne sont pas arpentées. Le seigneur fait arpenter un rang à la fois. Quand toutes les terres de ce rang sont concédées, "il en fait arpenter un autre, et les Concessionnaires s'adressent aussitôt que possible au Grand-Voyer pour l'ouverture des routes nécessaires". En général, les jeunes gens désirent s'établir sur de nouvelles terres. Aussi leurs parents ont-ils soin de s'en faire concéder une quantité suffisante, en vue d'y établir leurs garçons lorsque ceux-ci seront en âge de les cultiver ${ }^{98}$. Cela leur permet, entretemps, de commencer le défrichement de ces terres.

Dans le district de Montréal, une paroisse tranche sur les autres: celle de Saint-Benoît, sur la seigneurie des Deux-Montagnes. La première concession faite en ce lieu, datait de 1783 , ce qui explique le grand nombre des bonnes terres dont le seigneur disposait encore, soit environ deux cent cinquante. Elles n'étaient pas arpentées, il est vrai, ni les chemins tracés. Mais par contre, le taux était modique et les trois premières années exemptes de redevances. L'homme le plus pauvre trouvait le moyen de s'y établir sur une terre ${ }^{99}$.

Voilà les endroits où nous avons des preuves qu'il était aisé

97. Témoignage de John Macnider, Neilson et Cowan, éd., Journaux de la Chambre d'Assemblee du Bas-Canada, 32 (1823), Appendice T.

98. Témoignage de Joseph Badeaux, 8 décembre 1823, Appendice du XXXIIIe volume des Journaux de la Chambre d'Assemblée de la province du Bas-Canada, Appendice R, Appendice A; Letters from the Curates, 121-122.

99. Ibid., 69-70. 
d'obtenir des terres. Ces paroisses font nettement figure d'exceptions: les redevances seigneuriales y étaient modiques, quand un peu partout elles étaient à la hausse.

Ailleurs, il restait aussi des terres non concédées mais, en général, un obstacle quelconque s'opposait à leur mise en valeur. Du moins, il n'est pas dit que le defrichement y progressait.

Ainsi, il restait une couple de rangs de terres dans la seigneurie de Saint-Pierre les Becquets ${ }^{100}$. Etait-il possible d'en obtenir? a quelles conditions? nous l'ignorons.

Il en restait vingt-cinq mille arpents dans la paroisse de SainteMarie, comté de Nicolet. Une route y conduisait, la terre était propre à la culture mais non arpentée ${ }^{101}$. Si cette dernière expression signifie que le seigneur ne faisait arpenter qu'une terre à la fois, à mesure qu'il accordait une nouvelle concession - comme c'était, semble-t-il, l'usage général ${ }^{102}$, - cela ne nuisait peut-être pas au défrichement. Encore fallait-il le faire. N'est-il pas arrivé, à SaintJoseph de la Pointe-Lévi, que des concessionnaires ont été obligés "d'abandonner leurs Terres, après les avoir gardées et même cultivées pendant plusieurs années" 103 ? Mais si l'expression non arpentée signifie que les limites de la seigneurie n'étaient pas connues de façon précise, le seigneur ne pouvait faire aucune concession avant d'avoir fait recommencer l'arpentage de sa seigneurie. Ce travail était long et coûteux: Olivier Arcand, aidé de ses deux chaîneurs, prit "tout l'été des années 1820 et 1821", pour fixer le bornage des seigneuries Barrow et "diviser les fiefs Bonsecours et Bourgmarieouest en terres à culture" 104 . Ce travail requérait la coopération des seigneurs voisins: "je désirerais bien que nous puissions reouvrir la ligne seigneuriale...", écrivait le seigneur Tonnancour à l'agent de la seigneurie voisine, "il me devient indispensable de requerir

100. Témoignage de Joseph Badeaux, 8 décembre 1823, Appendice du XXXIIIe volume des Journaux de la Chambre d'Assemblee de la province du Bas-Canada, Appendice R, Appendice A.

101. Letters from the Curates, 100.

102. Témoignage d'Olivier Arcand, arpenteur, Appendice du XXXIIIe volume des Journaux de la Chambre d'Assemblee de la province du Bas-Canada, Appendice R, Appendice A.

103. Letters from the Curates, 44.

104. O.-M. H.-Lapalice, Histoire de la seigneurie Massue et de la paroisse de Saint-Aime (s. 1., 1930), 117. 
votre consentement... en conséquence je vous prirois bien de me faire une réponse aussitot que faire se pourra a ce sujet, ne pouvant faire aucune concession dans mon fief avant que la ditte ligne soit mise visible." Et si le voisin ne répondait pas ${ }^{105}$, ou si, mécontent du résultat, il engageait un autre arpenteur ${ }^{106}$ dont les calculs étaient différents de ceux du premier ${ }^{107}$, cela nuisait à l'agriculture.

Il restait des terres non arpentées, dépourvues de route, dans les paroisses suivantes:

Malbaie (un post-scriptum signale: soixante-quinze terres ont été concédées ces quinze derniers jours) ${ }^{108}$, Saint-Joseph de la PointeLévi, près de la paroisse ${ }^{109}$; Sainte-Croix ${ }^{110}$; Sainte-Elisabeth (comté de Joliette) ${ }^{111}$; Saint-Roch (comté de l'Assomption), dans la seigneurie Pangman seulement ${ }^{112}$; Vaudreuil, il en restait peu et elles semblaient réservées aux fils des anciennes familles ${ }^{113}$; Rigaud; ${ }^{114}$ et dans les seigneuries de Lotbinière et Deschaillons ${ }^{115}$.

Dans la paroisse de La Norraie, il restait de la terre, beaucoup en désiraient, mais il n'y avait pas de route pour s'y rendre ${ }^{116}$.

Le défaut de route, voilà un des principaux obstacles qui entravaient la marche du défricheur. "Il n'y a presque aucuns chemins à travers ces Terres non concédées, pas même pour y parvenir", affirme l'arpenteur Olivier Arcand, en parlant des comtés de Hampshire et de Buckinghamshire ${ }^{117}$. Un autre député, John Neilson,

105. Ibid., 115.

106. Ibid., 118.

107. Letters from the Curates, 24.

108. Ibid., 3.

109. Ibid., 44.

110. Ibid., 45; Témoignage d'Olivier Arcand, Appendice du XXXIIIe volume des Journaux de la Chambre d'Assemblé de la province du Bas-Canada, Appendice R, Appendice A.

111. Letters from the Curates, 55.

112. Ibid., 60.

113. Ibid., 72.

114. Ibid., 73.

115. Témoignage d'Olivier Arcand, Appendice du XXXIIIe volume des Journaux de la Chambre d'Assemblée de la province du Bas-Canada, Appendice R, Appendice A.

116. Letters from the Curates, 55.

117. Témoignage d'Olivier Arcand, Appendice du XXXIIIe volume des Journaux de la Chambre d'Assemblée de la province du Bas-Canada, Appendice R, Appendice $A$. 
parfaitement renseigné sur la question des chemins, pour s'être occupé activement, pendant plusieurs années, de colonisation agricole pouvait dire de la région de Québec: "Il n'y a de chemins sur aucune des terres non-concédées, excepté les chemins d'hiver, faits à l'arrière des établissements canadiens, pour transporter le bois ${ }^{118}$." "Les propriétaires fonciers, signalait le curé de Rouville, sont... obligés d'ouvrir toutes les routes, ou de payer leur part en prenant une concession, si le Seigneur les a fait ouvrir. Il n'y a rien de plus décourageant ${ }^{119}$." Le seigneur L. Juchereau-Duchesnay reconnaissait aussi que cela rebutait les colons et dépassait leurs moyens: "Les chemins sont en général demandés par les habitants qui occupent les rangs en profondeur... toujours la classe la plus pauvre", cela nuit considérablement au progrès du défrichage ${ }^{120}$. L'Honorable A.-L. J.-Duchesnay constatait: "pour obliger tous tenanciers à travailler à leurs chemins de même qu'aux routes publiques", la loi demande "d'appeler le Grand-Voyer du District". Or, "les Honoraires demandés par les Grands-Voyers et leurs frais de voyage sont très-hauts", ce qui occasionne "des dépenses au-dessus des moyens d'une grande partie d'entr'eux" ${ }^{\prime 21}$. L'agent Shadgett, qui avait entrepris la création d'un village anglais près du lac de Beauport, partageait l'opinion de son maître: "Les difficultés de cet Etablissement me paroissent venir entièrement du manque de chemins, ce qui seul a détourné de s'établir ceux qui ne l'étoient pas encore: ajoutez à cela les frais, la perte du tems et les formalités nécessaires pour avoir des Procès-Verbaux, avec la certitude d'oppositions de la part des anciens habitans situés sur la route de ces chemins." Certains individus les empêchent de passer sur leurs terres incultes, d'autres "ne veulent pas laisser abattre un arbre, quoique sur leurs terres à bois, pour passer en charrettes durant les mois d'été, à moins qu'il n'ait été obtenu un Procès-Verbal régulièrement homologué". Or cela coûte cher: "Un chemin de vingt arpens seulement, demandé pour donner aux gens établis une communication avec la ligne seigneuriale, a été obtenu, après beaucoup d'opposition de la

118. Témoignage de John Neilson, 31 décembre 1823, Ibid.

119. Letters from the Curates, 99.

120. Témoignage de L.-Juchereau Duchesnay, Neilson et Cowan, éd., Journaux de la Chambre d'Assemblée du Bas-Canada, 32 (1823), Appendice 'T.

121. Témoignage de l'Honorable A.-L.-J. Duchesnay, Ibid. 
part des habitans qui ont des Terres à bois et incultes, et a coûté à ces gens onze Louis deux shellings et demi [Eleven Pounds two shillings, and six Pence] d'honoraires au Grand-Voyer et à son Député." A la question: "Seroit-il difficile d'avoir encore des gens pour établir des terres, si vous en aviez de semblablement situées à leur donner aux mêmes taux"?, Shadgett répondit: "S'il y avoit des chemins d'ouverts pour communiquer avec chaque rangée, il n'y auroit aucune difficulté à établir quelque quantité que ce soit de terre semblablement située, et aux mêmes taux ${ }^{122}$." Puisque les honoraires des grands voyers étaient un sujet de plainte général, pourquoi n'étaient-ils pas portés au compte de la Province? A coup sûr, Papineau aurait rendu service à ses compatriotes s'il s'était employé à faire inscrire le nom de ces messieurs sur la liste civile.

Ailleurs, c'était le manque de eanaux d'irrigation, qui éloignait le défricheur. Il restait des terres difficiles à égoutter, dans les paroisses suivantes: Aux Cèdres, seigneurie de Soulanges, (il ne serait pas avantageux de s'y établir) ${ }^{123}$, Saint-Charles ${ }^{124}$, Saint-Hyacinthe, (le seigneur avait de plus augmenté les rentes) ${ }^{125}$.

Il restait des terres difficiles à égoutter, sans route et dont les rentes étaient élevées, dans les paroisses de Nicolet ${ }^{126}$, Saint-Michel, Yamaska ${ }^{127}$.

Dans cette dernière, le problème des routes se compliquait par suite de l'incertitude de la ligne de démarcation entre les districts de Trois-Rivières et de Montréal. Une route, commencée dans l'un avec le grand voyer de l'endroit, ne pouvait se continuer au delà de la ligne de démarcation, sans un procès-verbal du grand voyer de l'autre district. Comme on ne s'entendait pas sur la ligne en question, il restait six arpents de chemin à faire, mais aucun des deux hommes ne se croyait autorisé à l'homologuer ${ }^{128}$.

122. Témoignage de Shadgett, Ibid.

123. Letters from the Curates, 74.

124. Ibid., 101 .

125. Ibid., 103.

126. Ibid., 117; Témoignage de Joseph Badeaux, 8 décembre 1823, Appendice du XXXIIIe volume des Journaux de la Chambre d'Assemblée de la province du BasCanada, Appendice R, Appendice A.

127. Témoignage de Pierre-Basile Pelissier, Ibid.

128. Letters from the Curates, 113. 
A la paroisse de Saint-Michel, on peut joindre la seigneurie Barrow qui fut desservie jusqu'en 1824 par le curé d'Yamaska ${ }^{129}$. Cette seigneurie en comprenait cinq: Bourchemin, Bonsecours, Saint-Charles et Bourgmarie-ouest appartenant au capitaine Thomas James Barrow; et Bourgmarie-est, propriété de Margaret Barrow ${ }^{130}$. Pour y avoir une terre, il fallait entrer en relation avec l'agent, Samuel Gale, résidant à Montréal, lequel s'adressait ensuite aux héritiers Barrow résidant en Angleterre pour obtenir l'ordre de concéder la terre ${ }^{131}$. Un tel système pouvait difficilement fonctionner bien. Lapalice a compté "toutes les concessions que firent madame Barrow et ses héritiers, pendant la période qu'ils possédèrent la seigneurie. Le nombre se chiffre d environ soixante-douze, de 1790 a 1833"'132. Les bornes de ces seigneuries étaient mal connues, elles ne furent fixées définitivement qu'en $1821-22^{133}$. Les rentes y étaient trois fois plus considérables que sous le gouvernement français ${ }^{134}$. A la paroisse de Saint-Michel et aux seigneuries Barrow, joignons encore Bourchemin-est appartenant aux héritiers de Patrick Langan ${ }^{135}$ et les deux seigneuries de Wurtele: Bourgmarie-est et Déguire que les gens de l'époque englobaient dans leurs témoignages ${ }^{136}$. Beaucoup de personnes, avouait le notaire d'Yamaska, désirent prendre des terres dans les seigneuries Barrow, Deguire et Bourgmarie-est, mais elles "trouvent les taux trop chers, outre que ces terres sont très difficiles à égouter, et il faut des frais considérables

129. O.-M. H.-Isapalice, Histoire de la seigneurie Massue et de la paroisse de Saint-Aime, 112.

130. Ibid., 117.

131. Témoignage de Pierre-Joseph Chevrefils, notaire, 22 décembre 1823, Appendice du XXXIIIe volume des Journaux de la Chambre d'Assemblee de la Province du Bas-Canada, Appendice R, Appendice A.

132. O.-M. H.-Lapalice, Histoire de la seigneurie Massue et de la paroisse de Saint-Aime, 114.

133. Ibid., 118.

134. Témoignage de Pierre-Basile Pelissier, Appendice du XXXIIIe volume des Journaux de la Chambre d'Assemblé de la province du Bas-Canada, Appendice R, Appendice A.

135. O.-M. H.-Lapalice, Histoire de la seigneurie Massue et de la paroisse de Saint-Aime, 117.

136. Témoignage de Pierre-Joseph Chevrefils, 22 décembre 1823, et d'Olivier Arcand, Appendice du XXXIIIe volume des Journaux de la Chambre d'Assemblée de la Province du Bas-Canada, Appendice R, Appendice A. 
pour donner un cours à l'eau, et n'ayant aucun chemin pour y parvenir"'137. Plusieurs tentèrent l'expérience, ils en furent quittes pour leur peine; à preuve les saisies opérées en 1816 pour arrérages de rentes ${ }^{138}$, et les ventes de terres: la moitié des trois cents terres concédées dans la région entre 1810 et 1823, ont été revendues; un quart de celles qui ont été concédées au cours de 1822-23, sont déjà passées en d'autres mains. Le notaire prenait évidemment l'effet pour la cause lorsqu'il ajoutait: les jeunes gens de la région ne s'établissent pas sur des terres parce qu'ils "sont trop accoutumés de voyager dans les pays d'en haut". ${ }^{139}$

Il restait des terres, mais il n'y avait pas de routes et les rentes demandées étaient considérables, dans les paroisses de Cap-Sante $e^{140}$, Saint-André de Kamouraska ${ }^{141}$, Saint-Régis, les cultivateurs établis depuis plusieurs années n'avaient pas encore de route ${ }^{142}$, et dans les seigneuries de Gaudarville ${ }^{143}$, et de Beauport ${ }^{144}$.

Cette dernière, mesurant une lieue de front sur quatre de pro. fondeur, renfermait dans ses limites de grandes surfaces non concédées ${ }^{45}$. Le propriétaire, l'Honorable A.-L. J.-Duchesnay, en avait fait arpenter une certaine quantité en lots de soixante arpents carrés, que son agent concédait aux conditions suivantes: le censitaire, exempté de toute redevance pendant les trois premières années, devait ensuite payer annuellement cinq piastres de rentes plus six sols de cens et

137. Témoignage de Pierre-Joseph Chevrefils, notaire, 22 décembre 1823, Ibid.

138. O.-M. H.-Lapalice, Histoire de la seigneurie Massue et de la paroisse de Saint-Aimé, 119.

139. Tómoignage de Pierre-Joseph Chevrefils, 22 décembre 1823, Appendice du XXXIIIe volume des Journaux de la Chambre d'Assembles de la province du BasCanada, Appendice R, Appendice A.

140. Letters from the Curates, 13.

141. Ibid., 19.

142. Ibid., 82.

143. Témoignage de L. Juchereau Duchesnay, Neilson et Cowan Gd., Journaus de la Chambre d'Assemblée du Bas-Canada, 32 (1823), Appendice T; Témoignage de John Neilson, 31 décembre 1823, Appendice du XXXIIIe volume des Journaux de la Chambre d'Assemblee de la province du Bas-Canada, Appendice $\mathbf{R}$, Appendice A.

144. Témoignage de John Neilson, 31 décembre 1823, Ibid.; Témoignager de l'Honorable A.-L. J. Duchesnay et de Shadgett, Neilson et Cowan, Gd., Journaure de la Chambre d'Assemblee du Bas-Canada, 32 (1823), Appendice T.

145. Témoignage de l'Honorable A.-I. J. Duchesnay, Ibid. 
fournir une journée de corvée ${ }^{146}$. A l'époque, ce taux était considéré comme très élevé, surtout pour un terrain rocailleux et pauvre ${ }^{147}$. Le seigneur, qui avait tout intérêt à ne pas parler des rentes, insistait uniquement sur le coût élevé des travaux de voirie.

Il restait des terres médiocres, non arpentées, dépourvues de route, pour lesquelles on exigeait de fortes rentes, dans les paroisses de Sainte-Thérèse de Blainville, terrain sablonneux, rentes doublées, (à l'ancien taux, il n'en resterait aucune) ${ }^{148}$, Saint-Augustin, seigneurie de Faussembault ${ }^{149}$. Il restait des terres non arpentées, sans route et à rentes majorées, dans les paroisses de Saint-François de NouvelleBeauce, (terres de 3 arpents par 20) ${ }^{150}$, Sainte-Anne de la Pérade ${ }^{151}$, Saint-Henri de Mascouche ${ }^{152}$, Baie-du-Febvre, seigneurie Courval ${ }^{153}$.

Il ressort de cette enquête, que deux grands obstacles barraient la route au défricheur, là où des terres étaient encore disponibles: "les longs et côteux procédés pour faire ouvrir et homologuer des cours d'eaux si nécessaires dans la plus grande partie des Terres de l'intérieur"'154 et les honoraires considérables que les gens doivent payer en procès-verbaux pour avoir des chemins ${ }^{155}$. Par ailleurs, à l'ouverture de la session de 1820, le Gouverneur avait invité le Parlement provincial à encourager le défrichement des terres incultes, en faisant ouvrir de nouvelles routes. De plus, un des doyens

146. Témoignage de l'Honorable A.-L. J. Duchesnay, Ibid.

147. Témoignage de John Neilson, 31 décembre 1823, Appendice du $X X X I I I e$ volume des Journaux de la Chambre d'Assemblée de la province du Bas-Canada. Appendice R, Appendice A.

148. Letters from the Curates, 68.

149. Ibid., 11; Témoignage de L. Juchereau Duchesnay, Neilson et Cowan, éd., Journaux de la Chambre d'Assemblé du Bas-Canada, 32 (1823), Appendice T; Tómoignages de John Neilson, 31 décembre 1823, et d'Olivier Arcand, Appendice du XXXIIIe volume des Journaux de la Chambre d'Assemblee de la province du BasCanada, Appendice R, Appendice A.

150. Letters from the Curates, 40.

151. Ibid., 62.

152. Ibid., 112; Témoignage de Louis Gendron, 11 décembre 1823, Appendice du XXXIIIe volume des Journaux de la Chambre d'Assemblee de la province du BasCanada, Appendice R, Appendice A.

153. Témoignage d'Olivier Arcand, Ibid., Letters from the Curates, 116.

154. Témoignage d'Olivier Arcand, Appendice du XXXIIIe volume des Journaux de la Chambre d'Assemblée de la province du Bas-Canada, Appendice R, Appendice $A$.

155. Témoignage de John Neilson, 31 décembre 1823, Ibid.; Letters from the Curates, 13. 
du Conseil législatif, l'Honorable A.-L. J. Duchesnay, avait tendu la main à la Chambre basse et lui avait demandé de l'aide pour les travaux de voirie. Un peu de diplomatie n'aurait-elle pas inspiré à Papineau la conduite opportune? Est-ce que les députés n'auraient pas mieux compris leur rôle, s'ils avaient surmonté leur phobie de la dépense? Mais il était écrit qu'on ne gaspillerait pas le revenu de la Province!

\section{CHAPITRE IV}

\section{LES EXACTIONS DE CERTAINS SEIGNEURS}

Terres disponibles, mais taux exorbitant - Terres disponibles, mais à vendre - Terres disponibles, mais on refuse de les concéder - Les seigneuries du gouvernement - Consequences: subdivision des terres - Terrains empiétess.

Le jeune homme qui prenait une terre non défrichée, était moins que tout autre capable de payer une rente élevée, surtout s'il avait des dépenses spéciales pour travaux de voirie, si sa terre était de qualité médiocre ou difficile à égoutter ou de petites dimensions. Or, il appert que la hausse des taux s'ajoutait généralement, comme circonstance aggravante ou empêchement majeur, aux obstacles déjà mentionnés. Nombre de curés de paroisse attribuent à la hausse des taux de concession, le peu de progrès du défrichement à l'intérieur de la zone seigneuriale. Tous ont le même refrain: beaucoup de gens de cette paroisse prendraient des terres si les rentes n'étaient pas si élevées'.

L'exemple de la paroisse des Cèdres nous donnera une idée de la situation faite aux habitants par cette malencontreuse augmentation des cens et rentes. Dans cette partie de la seigneurie de Soulan-

1. Ce qui ne veut pas dire nécessairement que les rentes ont été augmentées dans leur paroisse, ni qu'il y reste des terres disponibles.

Letters from the Curates, 19, 21, 23, 26, 30, 36, 38, 40, 48, 51, 53, 58, 61, 68, 81, 82, $84,87,91,89,99,100,103,109,110,112,113,116,118,120$; Témoignage de Shadgett, Neilson et Cowan, éd., Journaux de la Chambre d'Assemblee du Bas-Canada, Appendice $\mathrm{T}$; Témoignages de Robert Jones, 8 décembre 1823, Louis Gendron, 11 décembre 1823, Pierre-Basile Pélissier, Pierre-Joseph Chevrefils, 22 décembre 1823, John Neilson, 31 décembre 1823, Appendice du XXXIIIe volume des Journaux de la Chambre d'Assemblee de la province du Bas-Canada, Appendice R, Appendice A. 
ges, les terres accordées par les dernières concessions étaient moitié plus petites que celles accordées avant la conquête et le taux deux fois plus élevé. La concurrence américaine se faisait sentir fortement: les barges des Etats-Unis nous apportent 24,600 barils de farine par semaine, durant toute la saison que le Fleuve est navigable, remarque le curé. Cela, joint au manque de bons moulins banaux dans la seigneurie, enlevait aux habitants tout espoir de vendre leur farine et tout désir de se procurer de meilleur blé de semence. Même le foin était rare dans la paroisse. Aussi, rien d'étonnant si les censitaires avaient peine à payer leurs rentes. Pour sa part, l'abbé Manseau jugeait le taux en vigueur excessif. Le résultat? Il est arrivé, raconte-t-il, que des gens incapables d'avoir des terres a meilleur terme, en ont acheté de censitaires de la seigneurie de Soulanges. Ces ventes ont produit des lods et ventes. Les acheteurs appauvris par la lourde rente annuelle, le coût de la propriété, les déboursés inévitables à tout début, ont été obligés de revendre la propriété hypothéquée. Nouveaux lods et ventes. Un second acheteur, à moins de le supposer riche, ne pouvait réussir mieux que le premier. Ainsi, les terres ont da changer de mains plusieurs fois, ou bien les propriétaires, s'ils ont voulu les conserver, ont da s'endetter pour la valeur de la propriétée . Les Cèdres était pourtant une vieille paroisse avantageusement située.

De Chambly, où il ne restait aucune terre, le curé écrivait: "la plupart des terres au-delà de Saint-Jean, auraient probablement été établies jusqu'à la frontière américaine, si les agents de ces seigneuries avaient suivi, ou à peu près, les pas des anciens seigneurs"3. Dans une seigneurie non loin d'ici, raconte le curé de Saint-Charles de Bellechasse, plusieurs personnes sont allees successivement voir le seigneur dans le but de se faire concéder une certaine étendue de bonne terre, mais toutes ont été rebutées par les conditions onéreuses qu'il posait. ${ }^{4}$. Ce qui retarde le défrichement des seigneuries, dit le curé de Rivière-Ouelle, c'est le taux élevé auquel les seigneurs font leurs concessions, en sorte que peu de gens peuvent en prendre a ces conditions. "Dans le Fief St-Denis, le Seigneur, dans ses nou-

2. Letters from the Curates, 75-76.

3. Ibid., 98.

4. Ibid., 38. 
velles Concessions, a de plus obligé le concessionnaire à payer une livre de sucre par dix livres qu'il fait ${ }^{5}$ ". Pourquoi, demande le curé de Saint-André de Kamouraska, exiger une certaine redevance en sucre d'érable, alors que le sucre constitue très souvent la seule source de revenu d'une nouvelle terre ${ }^{6}$ ?

L'augmentation des rentes a été suivie de résultats néfastes dans la Province. Les jeunes gens ne se mariaient pas, parce qu'ils n'avaient pas les moyens de prendre une terre. C'est ainsi que la paroisse de Saint-Gervais contenait, à l'époque, 350 hommes au-dessus de 21 ans, dont 10 seulement étaient cultivateurs propriétaires. Je ne sais pas très bien, avouait le curé, ce qui empêche les jeunes de prendre le peu de bonnes terres qui restent. Cependant on peut, en général, dire que c'est le taux trop élevé et les conditions onéreuses qu'on impose ${ }^{7}$. Les plus courageux bravaient la fatalité, mais la plupart d'entre eux, selon le curé de Saint-Jean Port-Joli, abandonnaient leur terre, incapables d'honorer les clauses de leur contrat $^{8}$. Ceux qui prennent des terres, corroborait le curé de SaintJean de Rouville, ne peuvent les garder longtemps et sont obligés de les remettre au seigneur. C'est plutôt rare, dans les seigneuries nouvellement concédées, que les tenanciers actuels aient été les premiers concessionnaires?.

Au dire de l'arpenteur Olivier Arcand, député de Hampshire, la situation était critique dans tout le comté qu'il représentait, c'est-adire dans toutes les seigneuries sises sur la rive nord entre SainteAnne de la Pérade et Cap-Rouge: dans Hampshire, beaucoup de personnes désireraient s'établir dans le voisinage de leurs parents et amis, mais "les Terres à concéder sont rares, et les rentes extraordinairement rehaussées;" on peut avoir des terres lorsqu'on est recommandé et qu'on aceepte de payer de fortes rentes; parfois, il faut même verser, avant la signature du contrat un droit de préférence $^{10}$. Un cultivateur de Sainte-Anne de la Pérade, Louis Gendron,

5. Ibid., 22.

6. Ibid., 19 .

7. Ibid., 32-39.

8. Ibid., 29.

9. Ibid., 99 .

10. Témoignage d'Olivier Arcand, Appendice du XXXIIIe volume des Journaux de la Chambre d'Assemblee de la province du Bas-Canada, Appendice R, Appendice A. 
confirmait le témoignage précédent par l'exposé de sa situation personnelle: l'ancien seigneur, monsieur de Lanaudière, demandait cher pour ses terres. Vu la nécessité où je me trouvais, j'en pris une il y a cinq ans. Mais "à cause des fortes rentes que j'avais de la peine à payer", j'ai été obligé de la revendre. Depuis un an et demi, j'ai une terre du seigneur actuel, monsieur Hale, au même taux mais je n'ai rien à payer pendant les cinq premières années. Je suis père de cinq garçons et de cinq filles. Mes garçons seraient d'âge à s'établir, mais la chose sera impossible à moins que je ne leur trouve des terres "à rentes modiques"11.

A Saint-Roch, comté de l'Assomption, le taux dans la seigneurie de Saint-Ours était de $2 \frac{1}{4}$ minots de ble plus une somme de 5 livres 4 sols ancien cours, pour 3 arpents par 30 . Dans la seigneurie de La Chesnay, appartenant à Peter Pangman ${ }^{12}$, il était de $41 / 2$ minots de blé plus 9 livres en argent, pour la même superficie. "Certains chefs de famille dans l'aisance ont été capables de prendre des Terres de M. Pangman à ce taux élevé; mais les ouvriers n'ont pas osé le faire, tandis qu'avec $M$. de St-Ours un grand nombre de serviteurs à gages ont été capables, à l'aide d'économies faites sur leur salaire, de devenir ce qu'ils sont aujourd'hui des cultivateurs dans l'aisance". Plusieurs garçons, établis par leurs parents dans la seigneurie Pangman, n'ont pas été capables de s'y maintenir. A l'ancien taux, environ deux cents jeunes hommes prendraient des terres ${ }^{13}$.

Dans la seigneurie de Lanaudière, propriété de M. Pothier, le taux des rentes est si exorbitant, affirmait le député de TroisRivières, que très peu osent $\mathrm{y}$ prendre une concession. Beaucoup de jeunes gens des paroisses de Maskinongé, Machiche et de la Rivièredu-Loup ont été obligés de traverser le Fleuve pour avoir des terres. Une centaine de familles se sont établies dans le voisinage de la seigneurie de la Rivière David sur le Township d'Upton ${ }^{14}$. - A partir de 1822, plusieurs se dirigèrent vers la seigneurie Barrow ${ }^{15}$.

11. Témoignage de Louis Gendron, 11 décembre 1823, Ibid.

12. "Minutes du Conseil exécutif", 25 novembre 1803, APC, Q 2, 21-22.

13. Letters from the Curates, 60-61.

14. Témoignage de Joseph Badeaux, 8 décembre 1823, Appendice du XXXIIIe volume des Journaux de la Chambre d'Assemblée de la province du Bas-Canada, Appendice R, Appendice A.

15. O.-M. H.-Lapalice, Histoire de la seigneurie Massue et de la paroisse de Saint-Aimé, 118. 
Je n'ai aucun doute, écrivait l'abbé Leduc, que si on ramenait les rentes à l'ancien taux, des deux cents terres qui ne sont pas encore concédées dans Saint-Polycarpe (seigneurie de Nouvelle-Longueuil), il n'en resterait aucune au bout de l'année. Mais les rentes sont oppressives: le seigneur exige au moins huit sols de rente par arpent carré, plus six piastres pour le titre et sept autres piastres pour la concession; la terre qu'il cède à ce prix, n'est que de trois arpents par vingt: Celui qui s'aventurerait à prendre une terre dans ces conditions ne pourrait la garder, affirmait le curé. Même ceux qui sont déjà établis et paient moins cher de rentes, sont dans la gêne. Sur 240 cultivateurs, à peine 30 n'achètent pas de blé; "les terres étant si courtes, les cultivateurs resteront probablement toujours pauvres, malgré la fertilité du sol, parce qu'il est nécessaire qu'ils se gardent du bois". Malgré leurs demandes réitérées, aucun d'eux n'a pu obtenir une étendue additionnelle de terre, "et pourquoi? Parce qu'entretemps le Seigneur vend tout le Pin et le Chêne à des personnes qui ouvrent des Cours à Bois". Sur les terres arpentées cette année, tout le bois utile a été vendu par le seigneur. "Si nos pauvres cultivateurs se plaignent, ce n'est pas sans raison." Etant dans l'impossibilité de s'établir, nos jeunes vont s'épuiser dans les Pays d'en haut. Ils nous reviennent ensuite avec une constitution ruinée et sont un grand fardeau pour la sociététe.

En augmentant les redevances, les seigneurs violaient une des clauses de leur titre de concession. Pour s'en convaincre, le Comité des Terres de la Couronne fit examiner quelques-uns de ces écrits et tira la conclusion suivante: "En référant à ces Concessions on trouvera que la condition de toutes étoit de concéder les Terres aux Habitans du Pays aux Rentes accoutumées ${ }^{17}$." L'augmentation des rentes était contraire à l'usage établi sous le roi français: "Avant la conquête, la tenure en fief au Canada, ne donnait guère, au Seigneur, plus que la prééminence et la surintendance dans la concession de ses terres à de vrais colons; pour ce travail il ne recevait que ce qui lui était dû pour son investissement de capital et les services qu'il rendait ainsi... Le censitaire ne pouvait pas, en vertu de la tenure, être

16. Letters from the Curates, 79-81.

17. A. Stuart, "Troisième rapport", 1821, dans Premier rapport, 2: 1. 
soumis à des fardeaux qu'il ne pouvait aisément porter"18. La hausse des taux de concession, contrevenait aux arrêts de Marly, lesquels interdisaient "aux seigneurs d'exiger des censitaires plus qu'on ne paie dans la région où est située la seigneurie" ${ }^{19}$. Un jugement du 23 janvier 1738 avait, par ailleurs, fixé les cens et rentes pour une terre de trois arpents par trente ${ }^{20}$ : "Dans le 30me volume fol 41 Ro des régistres des ordonnances et jugemens des intendans, il se trouve dans une contestation entre le seigneur de Gaudarville et ses censitaires, que ceux-ci doivent prendre des contrats de concessions "aux cens et rentes ordonnés par Sa Majesté, savoir un sol de cens par chaque arpent de front et un sol de rentes par chaque arpent en superficie, et un chapon ou vingt sols au choix du seigneur pour chaque arpent de front". Dans le 35me volumes fol $10 \mathrm{Ro}$, les cens et rentes ordinaires sont fixés à 1 sol pour chaque arpent en superficie, trois chapons pour toute une terre et 2 sols de cens ${ }^{21}$."

Les paroisses où il restait des terres disponibles, mais où les rentes étaient exorbitantes au point que personne n'en pouvait prendre à ces conditions, sont les suivantes:

Saint-Michel, (seigneurie près de la paroisse ${ }^{22}$ ), Saint-Henri de Lauzon, (pas de routes non plus ${ }^{23}$ ), Saint-Polycarpe, seigneurie de Nouvelle-Longueuil ${ }^{24}$, Saint-Luc, seigneurie Bleury et baronnie de Longueuil $^{25}$, Saint-Léon ${ }^{26}$, Yamachiche ${ }^{27}$, Bécancour ${ }^{28}$.

18. Témoignage de John Neilson, 31 décembre 1823, Appendice du XXXIIIe volume des Journaux de la Chambre d'Assemblee de la province du Bas-Canada, Appendice R, Appendice A.

19. G. Frégault, La Civilisation de la Nouvelle-France (Montréal, 1944), 191.

20. "un sol de cens par chaque arpent de front, et un sol de rente par chaque arpent en superficie, et un chapon, ou vingt sols, au choix du Seigneur, pour chaque arpent de front." Témoignage de John Neilson, 31 décembre 1823, Appendice $d u$ $X X X I I I e$ volume des Journaux de la Chambre d'Assemblee de la province du BasCanada, Appendice R, Appendice A.

21. Jean-Paul, "Lettres seigneuriales à Agricola", La Minerve, 30 mars 1837.

22. Ibid., 33.

23. Ibid., 43.

24. Ibid., 81.

25. Ibid., 91.

26. Ibid., 109.

27. Ibid., 113; Témoignage de Joseph Badeaux, 8 décembre 1823, Appendice du XXXIIIe volume des Journaux de la Chambre d'Assemblée de la province du BasCanada, Appendice R, Appendice A.

28. Letters from the Curates, 120. 
Ailleurs, on ne veut rien dire, mais ce silence même dénote une situation anormale. Le curé de Deschambault a consulté ses paroissiens pour savoir ce qu'il devait répondre: "quelques-uns ont peur de se créer des ennemis; d'autres ne veulent pas appuyer leurs assertions de leur signature"29. Cette seigneurie appartenait à Louis de la Gorgendière, Ecuyer, et à l'Honorable Juchereau-Duchesnay ${ }^{30}$.

Les seigneurs qui demandaient des rentes exorbitantes avaient, semble-t-il, l'arrière-pensée de garder leurs terres pour les vendre. On déplorait, en effet, que l'habitude commençât à s'implanter de vendre les terres au lieu de les concéder. Plusieurs curés dénoncent ces ventes faites secrètement avant de signer l'acte de concession, sans toutefois spécifier si ces exactions se pratiquaient dans leur paroisse ou ailleurs: ceux de Saint-Michel ${ }^{31}$, Saint-Joseph de la Pointe-Lévi ${ }^{32}$, Rivière-du-Loup, (en haut) ${ }^{33}$, Yamachiche ${ }^{34}$, SaintFrançois du $\mathrm{Lac}^{35}$.

Sur cet item, les témoignages sont plutôt réticents. Personne ne voulait se causer d'ennuis avec des affirmations qu'il aurait pu être mis en demeure de prouver ensuite; et le moyen de le faire, puisque ces ventes se faisaient sans témoin.

D'autres personnes dignes de confiance, avaient aussi entendu parler de ces ventes illicites, tels Joseph Badeaux, agent pour les biens des Jésuites du district de Trois-Rivières et seigneur du fief Courval $^{36}$, et le notaire Michel Berthelot, agent pour les biens des Jésuites du district de Québec ${ }^{37}$. Robert Jones affirmait avoir entendu dire que certains seigneurs du district de Montréal, offraient leurs terres non

29. Ibid., 14.

30. J. Bouchette, Description Topographique de la province du Bas-Canada, avec des remarques sur le Haut Canada, et sur les relations des deux provinces avec Les Etats Unis de l'Amérique, 393.

31. Letters from the Curates, 33.

32. Ibid., 44.

33. Ibid., 110.

34. Ibid., 113.

35. Ibid., 116.

36. Témoignage de Joseph Badeaux, 8 décembre 1823, Appendice du XXXIIIe volume des Journaux de la Chambre d'Assemblée de la province du Bas-Canada, Appendice R, Appendice A.

37. Témoignage de Michel Berthelot, notaire, 9 décembre 1823, Ibid., 
concédées à un prix aussi respectable que "dix piastres l'arpent"38. Le curé de Beaumont tenait d'un confident très fiable et parfaitement renseigné, qu'un seigneur des environs avait extorqué vingt piastres à un cultivateur, avant de lui concéder une terre ${ }^{39}$.

L'abbé Demers, curé de Saint-Luc, comté de Saint-Jean, parlant de la seigneurie Bleury et de la baronnie de Longueuil, dit que les terres y sont vendues ou concédées à si haut prix, que les gens sont incapables de les payer ${ }^{40}$.

"Seuls, dit le curé d'Yamachiche, les fils dont les parents sont dans l'aisance, peuvent s'établir. Les autres jeunes hommes qui seraient à peine en mesure d'acquitter les rentes d'une terre et qui sont a plus forte raison incapables d'en acheter une, s'engagent pour les Pays d'en haut, ou travaillent ici et là, puis marient des filles de rien, et sont incapables d'élever leurs enfants et sont finalement réduits à la mendicitét1."

Le seigneur canadien n'avait absolument pas le droit, - les arrêts de Marly étaient bien clairs sur ce point ${ }^{42}$ - de vendre ses terres non concédées. Il était tenu de les concéder et, sur son refus de le faire, le postulateur pouvait obtenir de la couronne, aux conditions ordinaires, la terre demandée. En ce cas, les revenus auraient été au profit du roi ${ }^{43}$. Sous le régime français, il suffit que la loi existât pour tenir les seigneurs dans la modération ${ }^{44}$. Après 1760 , à mesure qu'ils s'aperçurent du manque de vigilance des autorités anglaises en ce qui touchait à l'application des lois françaises, les seigneurs commencèrent à les transgresser et à s'adonner à la spéculation.

Certains poussèrent le défi à la loi jusqu'à refuser de concéder leurs terres incultes. Les curés de Saint-Régis ${ }^{45}$, de Saint-Léon (de

38. Témoignage de Robert Jones, 8 décembre 1823, Ibid.

39. Letters from the Curates, 36.

40. Ibid., 91.

41. Ibid., 113.

42. G. Frégault, La Civilisation de la Nouvelle-France, 190; A. Stuart, "Troisième rapport, 1821, dans Premier rapport, 2: 9-37; [ Anonyme ], "Notes sur les tenures dans le Bas-Canada", [ s.d., 1822 ], APC, Q 163-1: 119.

43. F.-J. Cugnet, Traité de la loi des fiefs (Québec, 1775), 60.

44. J. Bouchette, The British Dominions in North America; or a Topographical and Statistical Description of the Provinces of Lower and Upper Canada, New Brunswick, Nova Scotia, the Islands of Newfoundland, Prince Edward, and Cape Breton, 1: 378-379. 45. Letters from the Curates, 82. 
Maskinongé $e^{46}$ ), et de Saint-Michel (de Bellechasse, seigneurie Saint-Michel ${ }^{47}$ ), affirmèrent connaître de ces seigneurs qui violaient ainsi le premier mot des Arrêts de $1711^{48}$. Dans les paroisses suivantes, quoique le seigneur eût encore de la terre disponible, il refusait de faire de nouvelles concessions:

Grondines ${ }^{49}$, Saint-Michel,une seigneurie près de la paroisse ${ }^{50}$, Maskinongé, seigneurie de Lanaudière ${ }^{51}$.

Cette dernière, qui mesurait trois ou quatre lieues de profondeur, n'était pas concédée, sauf le fief Mary Anne. Le propriétaire, Pothier ${ }^{52}$, trouvait son profit dans la fabrication du sucre d'érable et la coupe du bois. Résultat: les jeunes s'engagent pour la Compagnie du Nord-Ouest; quand ils reviennent des Pays d'en haut, ils sont enclins à l'oisiveté et à l'ivrognerie, et ne deviennent jamais de bons cultivateurs.

A Sainte-Anne des Plaines, il reste peu de bonnes terres, écrivait le curé Grenier, elles ne sont pas arpentées, il n'y a pas de route, pour s'y rendre. Plusieurs fois, les seigneurs ont refusé de faire de nouvelles concessions. Aussi mes paroissiens ont-ils vu a vec étonnement, en 1820, soixante à quatre-vingts familles écossaises venir occuper les terres non concédées de la seigneurie de Laplaine. "Quoi! disentils, notre Paroisse est tout à fait récente; il y a quarante ans elle contenait très peu de colons; elle s'est peuplée rapidement; les Seigneurs n'ont que très peu de bonnes terres à concéder, pourquoi ne les réserveraient-ils pas pour nos enfants? Puisque à force de travail nous avons défriché la plus grande partie de leurs Seigneuries, ne semble-t-il pas juste que les continuations de nos propriétés soient concédées à nos enfants ${ }^{53}$ ?"

46. Ibid., 109.

47. Ibid., 33.

48. G. Frégault, La Civilisation de la Nouvelle-France, 190.

49. Témoignage d'Olivier Arcand, Appendice du XXXIIIe volume des Journaux de la Chambre d'Assemblée de la province du Bas-Canada, Appendice R, Appendice A; Letters from the Curates, 14.

50. Ibid., 33.

51. Ibid., 108.

52. Témoignage de Joseph Badeaux, 8 décembre 1823, Appendice du XXXIIIe volume des Journaux de la Chambre d'Assemblée de la province du Bas-Canada, Appendice R, Appendice A.

53. Letters from the Curates, 66. 
Dans les paroisses sises sur et autour de la seigneurie de Beauharnois, dont le propriétaire résidait à Londres, la situation était plus critique encore; la spéculation sur les terres y prenait une tournure de calamité. A Saint-Constant, par exemple: Ici, écrit le curé, toutes les terres sont prises. La seigneurie de Beauharnois est là tout près qui pourrait recevoir le surplus de population de cette paroisse, mais toujours Ellice s'y refuse. Ne pouvant obtenir de terre, beaucoup de jeunes font le voyage des Pays d'en haut pendant l'été et mènent ensuite une vie licencieuse pendant l'hiver, dépensant leur argent dans les tavernes ${ }^{54}$.

Situation analogue à Châteauguay: Toute la seigneurie était concédée. Beaucoup de gens désiraient prendre des terres dans celle de Beauharnois, mais le seigneur de cet endroit demandait trop cher ou refusait ${ }^{55}$.

L'abbé Manseau, curé aux Cèdres, faisait la constatation suivante: "Il y a, dans la seigneurie de Soulanges, une étendue de terre qui n'est pas encore concédée ou arpentée, qui formerait une concession de 28 terres de 3 arpens par 20 et 25 de profondeur. Aucune route n'a encore été ouverte à travers cette étendue de terre, le sol est de qualité moyenne et serait difficile à égoutter... Je ne vois pas que les jeunes hommes de Soulanges puissent tenter de s'établir avec avantage ailleurs que dans la Seigneurie de Beauharnois ou celle d'Annfield, appartenant à M. Ellice." Les dimensions de cette propriété sont de six lieues carrées; mais à peine un quart de cette surface est concédé. "Il ne semble pas que le reste de cette vaste étendue de terre ait jamais été arpenté, ou qu'on ait jamais pensé à y faire des chemins." Les gens qui ont tenté d'y prendre des terres ont été rebutés. "Il suffit d'être un instant sur les lieux et d'entendre les plaintes des habitants de cette seigneurie [Soulanges] pour réaliser combien la génération actuelle dans les environs souffre du refus obstiné du seigneur de Beauharnois, pendant près de vingt ans, de faire de nouvelles concessions... si le seigneur de ce Domaine [ Beauharnois] se décidait ou était obligé à faire de nouvelles concessions, on verrait, en quelques années, des centaines de familles s'établir là... y vivre dans une grande prospérité et bénir à jamais

54. Ibid., 89.

55. Ibid., 84. 
la mémoire des bienfaiteurs qui ont opéré ce nouvel état de choses ${ }^{56}$." Le contraindre! chose plus facile à dire qu'à faire, car on s'attaquerait ainsi au "très honorable Edward Ellice", dont on a pu écrire quelques années plus tard (1830): "Sa longue carrière parlementaire et sa parenté avec le comte Grey, qui était son beau-frère, ajoutait à peine à l'influence que lui assuraient sa grande fortune et ses talents incontestés ${ }^{57}$."

Le même prêtre, qui desservait aussi Saint-Timothée, ếcrivait de cette paroisse: C'est surtout ici que le désir d'avoir des terres de la seigneurie de Beauharnois, se fait sentir. Je connais une maison où sont installées, côte à côte, trois jeunes familles: bientôt ce sera la misère. Mais toujours le seigneur refuse d'accorder de nouvelles concessions ${ }^{58}$.

Quant aux gens déjà établis à Beauharnois, certains versaient une rente annuelle de 5 livres Sterling pour une terre de cinq arpents par quarante. Cela peut nous paraître peu de chose aujourd'hui. L'abbé Clément, pour sa part, déclarait ce taux exorbitant. Par ailleurs, l'agent n'était pas toujours, à ce qu'il parait, d'humeur commode: "Un tiers des gens n'ont pas leur titre, et ne peuvent pas se le procurer. La plupart d'entre eux sont surchargés d'arrérages de rentes depuis je ne sais combien d'années". Il sont disposés à payer; ils viennent offrir leur blé. A un moment, il n'a pas le temps; à un autre, il exige une certaine quantité additionnelle pour compenser la diminution de volume que subira le grain en séchant, par exemple un quart, un tiers de minot et même un demi minot par sac, ce qui est "certainement excessif; car, si nous pouvons en juger par le blé de dîme, qui est généralement le plus mauvais, deux poignées par sac sont bien suffisantes. Un certain nombre de terres ont ainsi été reprises par retrait, à cause des rentes, qu'il ne veut jamais accepter a temps. On dit... qu'il refuse de faire des concessions de façon à vendre ces terres ${ }^{59}$." Mais pendant qu'Ellice préférait attendre l'heure de faire un coup d'argent, son agent, sur les lieux commen-

56. Ibid., 74-75.

57. E. Bouchette, Mémoires de Robert-S.-M. Bouchette 1805-1840 Recueillis par son fils Errol Bouchette et Annotes par A.-D. Decelles (Montréal, 1903), 23.

58. Letters from the Curates, 78.

56. Ibid., 85-86. 
çait à se laisser fléchir: "Je vous prie instamment, lui écrivait-il, de me faire savoir si je puis ou non faire des concessions, car je m'attends à être tourmenté tout l'été par des solliciteurs. Si je refuse, un grand nombre, comme cela s'est produit par le passé, s'assoiront là et attendront. Les éloigner serait peine perdue, car un groupe ne sera pas sitôt parti, qu'un autre prendra sa place. La question est de savoir si, lorsqu'on ne peut pas faire disparaître un torrent, il n'est pas préférable de l'utiliser en dirigeant son cours dans un bief". Il serait bien à souhaiter que vous veniez vous-même sur les lieux. Votre présence ici, de si courte durée soit-elle, aurait des conséquences inappréciables au point de vue de vos propres intérêts ${ }^{60}$.

Du temps du roi français et de son intendant, les seigneurs étaient surveillés en ce qui concerne l'obligation de concéder leurs terres - le deuxième volume des Edits et Ordonnances est là pour attester certaines confiscations de fiefs non peuplés ${ }^{61}$. On ne souffrait pas que les Arrêts restassent lettre morte. Mais sous l'administration anglaise, les seigneuries du gouvernement étaient les premières a donner le mauvais exemple et à enfreindre les lois.

Dans Sorel, seigneurie de la couronne, il restait environ soixante terres de trois arpents par vingt, propres à la culture, mais personne ne pouvait en obtenir ${ }^{62}$. Bureaucratie? Incurie de rond-de-cuir? "Probablement, avouait Robert Jones, que le Gouvernement ignore qu'il lui reste des terres à concéder. D'ailleurs il n'a pas de plan de sa seigneurie ${ }^{63}$."

Cela n'est rien, cependant, comparé à la conduite stupide des commissaires préposés à l'entretien des biens des Jésuites. Ils a vaient le pouvoir des clés sur d'immenses territoires, mais s'en constituaient les cerbères et prenaient plaisir à refuser toute concession. Le notaire Michel Berthelot, qui avait été, pendant une vingtaine d'années,

60. John Richardson à Edward Ellice, 16 juin 1823, APC, Q 167-2: 384-385.

61. Témoignage de John Neilson, 31 décembre 1823, Appendice du $X X X I I I_{6}$ volume des Journaux de la Chambre d'Assemblee de la province du Bas-Canada, Appendice R, Appendice A.

62. Letters from the Curates, 105.

63. Témoignage de Robert Jones, 8 décembre 1823, Appendice du XXXIIIe volume des Journaux de la Chambre d'Assemblee de la province du Bas-Canada, Appendice R, Appendice A; O.-M. H.-Lapalice, Histoire de la seigneurie Massue et de la paroisse de Saint-Aime, 117-118. 
agent pour les biens des Jésuites du district de Québec, faisait la déposition suivante devant le Comité des Terres de la Couronne: Je n'ai jamais eu ni plans, ni titres originaux de ces propriétés. Je ne sais de quelle étendue elles sont. Je crois pourtant qu'il reste des terres dans les seigneuries Saint-Gabriel et Bélair. [ Il en restait effectivement dans Bélair; elles étaient de qualité médiocre $\left.{ }^{64}\right]$. J'ai fait des concessions à six personnes depuis que je suis agent. [ Six concessions en vingt ans, alors que, tout autour on se plaignait de ne pouvoir obtenir de terre!] Il m'aurait fallu un an pour mettre les papiers en ordre. J'ai demandé l'autorisation de faire ce travail; elle m'a été refusée ${ }^{65}$.

Dans le district de Trois-Rivières, la Commission des Biens des Jésuites administrait, entre autres, les deux immenses seigneuries du Cap-de-la-Magdelaine (3 lieues de front par 20 lieues de profondeur et de Batiscan 123/4 lieues de front par 20 lieues de profondeur). On disait, à l'époque, qu'un seizième seulement de la première était concédé $\hat{e}^{66}$ et que la seconde contenait environ cinq cents bonnes terres planches non concédées ${ }^{67}$. Quant à celle du Cap-de-la-Magdelaine, c'était un demi-mal, les terres sablonneuses de cette seigneurie étant, pour la plupart, impropres à la culture ${ }^{68}$. "Après beaucoup de demandes pour les deux seigneuries, révélait l'agent, j'ai eu la permission de concéder en 1809, à 24 francs de rente annuelle pour une terre de 3 arpents par 30. Comme c'était cher je n'en concédai que très peu"69. Au taux ordinaire, affirmaient des témoins mandés exprès de Saint-Stanislas, trois cents des terres non concédées de la seigneurie de Batiscan, le seraient à l'heure actuelle. Mais, à quatre piastres de rente annuelle, les jeunes sont

64. Letters from the Curates, 11.

65. Témoignage de Michel Berthelot, notaire, 9 décembre 1823, Appendice du $X X X I I I$ e volume des Journaux de la Chambre d'Assemblee de la province du BasCanada, Appendice R, Appendice A.

66. Témoignage de Joseph Badeaux, agent, 6 décembre 1823, Ibid.

67. Témoignages de Joseph Trépanier, François Ayotte, Pierre Trépanier, 6 décembre 1823, Ibid.

68. Témoignage de Wm Marchant, 15 décembre 1823, Ibid.; Letters from the Curates, 106.

69. Témoignage de Joseph Badeaux, 6 décembre 1823, Appendice du XXXIIIe volume des Journaux de la Chambre d'Assemblée de la province du Bas-Canada, Appendice R, Appendice A. 
dans l'impossibilité de prendre une terre et de fonder un foyer ${ }^{70}$. Beaucoup sont parvenus à l'âge adulte et ils attendent encore, pour s'établir, que les rentes demandées soient moins considérables ${ }^{71}$. Ils auraient pu attendre encore longtemps, comme on put s'en rendre compte par la suite de la déposition de l'agent: "Depuis 1817 ou 1818 je n'ai plus la permission de faire de concessions"'72. Les membres qui composaient la Commission, lorsqu'elle porta les rentes à quatre dollars par terre, étaient: Dunn, Baby, Williams, Berthelot et Pyke. Lorsqu'elle défendit de faire d'autres concessions, les commissaires étaient Ryland, W. Smith, Coltman, John Stewart, Foy ${ }^{73}$. Pendant que messieurs les Commissaires haussaient le taux des nouvelles concessions, puis refusaient des terres aux jeunes, ils n'entretenaient même pas l'ancien moulin banal des Jésuites. Depuis quinze ans, il n'était plus serviable et les cultivateurs de SaintStanislas devaient aller faire moudre leur grain à Sainte-Anne de la Chevrotière et même à Jacques-Cartier ${ }^{74}$.

Quand un père de famille se trouvait dans l'impossibilité de procurer une terre à ses garçons, il devait se résigner à les laisser partir pour les Pays d'en haut ou à partager sa terre avec un ou deux de ses fils. Au cap-de-la-Magdelaine, quelques terres sont divisées ${ }^{75}$. François Ayotte de Saint-Stanislas connaît trois familles qui ont divisé leur terre pour leur garçon et en sont dans la pauvreté ${ }^{76}$. La division des terres, dit-il, appauvrit la paroisse: le bois devient rare et la terre moins productive. Sur certaines vieilles terres, il n'y a plus de bois de construction et peu de bois de chauffage ${ }^{77}$. A SainteAnne de la Pérade, une terre de cinq arpents par quarante est divisée en trois, et une quarantaine d'autres familles ont partagé leur terre.

70. Témoignages de Joseph Trépanier, François Ayotte, Pierre Trépanier, 9 décembre 1823, Ibid.

71. Témoignage de Romain Mongrain, 6 décembre 1823, Ibid.

72. Témoignage de Joseph Badeaux, 6 décembre 1823, Ibid.

73. Témoignage de Joseph Badeaux, 8 décembre 1823, Ibid.

74. Témoignages de Joseph Trépanier, François Ayotte, Pierre Trépanier, 6 décembre 1823 , Ibid.

75. Témoignage de Wm Marchant, 15 décembre 1823, Ibid.

76. Témoignage de François Ayotte, 6 décembre 1823, Ibid.

77. Témoignages de Pierre Trépanier et James Lambert, 6 décembre 1823, Ibid. 
"Cela occasionne de la pauvreté et de la misère"78. J.-B. Proulx, député de Buckinghamshire, affirme que dans son comté, il se fait un grand nombre de subdivisions de terres ${ }^{79}$. Dans le comté de Hampshire, on subdivise les terres pour y établir plusieurs familles, affirme le député Olivier Arcand ${ }^{80}$. Dans la région de Québec, c'est pire encore, affirme John Neilson. Presque toutes les concessions ont été subdivisées en bandes étroites, dont quelques-unes sont à peine plus larges qu'une route ordinaire. Le long de la grand'route, les maisons sont si nombreuses qu'on dirait plutôt une rue de plusieurs milles de longueur. "Ces subdivisions, lorsqu'elles sont poussées jusqu'à l'excès que j'ai mentionné, produisent la pauvreté et la mendicité et leurs conséquences sont la dégradation et l'immoralité du peuple ${ }^{81}$." Les statistiques dressées par la Chambre, prouvent qu'il y avait des milliers de terres plus petites que 3 arpents par 30 et des centaines d'une surface moindre que 2 arpents par 30 . Des centaines ne mesuraient même pas un arpent par 30. Dans deux paroisses notamment, la plupart des terres avaient moins d'un arpent par trente de superficie ${ }^{82}$. Si l'on tient compte des méthodes de culture routinières usitées à l'époque, ces chiffres signifient des milliers de familles dans le dénuement complet.

Subdiviser les terres était préjudiciable au pays. Aussi une ordonnance du roi français, datée du 28 avril 1745 et enregistrée dans la Province ${ }^{83}$, défendait-elle formellement aux censitaires de bâtir maisons ou étables sur des terres mesurant moins d'un arpent et demi de front, par trente ou quarante de profondeur. Toute infraction à cette loi était passible d'une amende de cent livres tournois applicable au soulagement des familles les plus pauvres de l'endroit, et la maison ou l'étable devaient être démolies. Seule une grange destinée à abriter les récoltes, était tolérée sur une moindre étendue de terre, encore devait-elle être exclusivement en bois ${ }^{84}$. Sous le

78. Témoignage de Louis Gendron, 11 décembre 1823, Ibid.

79. Témoignage de J.-B. Proulx, 9 décembre 1823, Ibid.

80. Témoignage d'Olivier Arcand, Ibid.

81. Témoignage de John Neilson, 31 décembre 1823, Ibid.

82. J. Neilson, éd., Journaux de la Chambre d'Assemblée du Bas-Canada, 31 (1822), Appendice N.

83. Edits et Ordonnances, vol. i: 551.

84. F.-J. Cugnet, Traité de la loi des fiefs, 60-61. 
régime français, cette loi fut appliquée avec rigueur et c'est heureux. Après la Conquête... "Cependant, on s'en est éloigné imperceptiblement, et la condition de l'habitant dans la plupart des seigneuries en a été diminuée, l'accroissement du défrichage n'ayant pas marché de pair avec l'augmentation de la population ${ }^{85}$." On peut trouver une cause à ces maux, affirmait John Neilson, dans le manque d'éducation des gens de la campagne. Seule, en effet, une bonne éducation "pourrait les qualifier pour un autre emploi, et leur donner le désir et l'initiative de quitter leurs parents et amis pour chercher fortune au loin ${ }^{86} . "$

Il s'en trouva cependant qui payèrent d'audace et répondirent à l'illégalité par l'illégalité. Si les seigneurs ne sont pas tenus de respecter la loi, pourquoi le serions-nous davantage? L'enquête révéla que la propriété privée était violée en certains endroits et, qu'en d'autres, les terres de la couronne étaient envahies par des gens sans titre ${ }^{87}$. Profitant de ce que les propriétaires de la seigneurie Barrow demeuraient en Angleterre, une quinzaine de familles s'installèrent sans permission sur cette propriété. Certaines y étaient depuis quinze ans déjà, au moment de l'enquête, et n'en avaient jamais été inquiétées ${ }^{88}$. A Saint-Stanislas, sur la seigneurie de Batiscan, les jeunes, ne pouvant avoir de concessions, commencèrent à défricher, sans permission, des étendues de terre qu'ils se taillaient sur les parties non concédées de la seigneurie du gouvernement ${ }^{89}$. Une trentaine de jeunes gens cultivaient ainsi du blé et des pommes de terre, sur ces terres vacantes qu'on s'obstinait à leur refuser ${ }^{90}$.

Un simple mortel jouait même au seigneur depuis une vingtaine d'années, exerçant les droits seigneuriaux sur des terres qui ne lui ap-

85. J. Bouchette, The British Dominions in North America; or a Topographicaand Statistical Description of the Provinces of Lower and Upper Canada, New Brunswick, Nova Scotia, the Islands of Newfoundland, Prince Edward, and Cape Breton 1: 379 .

86. Témoignage de John Neilson, 31 décembre 1823, Appendice du XXXIIIe volume des Journaux de la Chambre d'Assemblée de la province du Bas-Canada, Appendice R, Appendice A.

87. A. Stuart, "Rapport", 3 février 1824, Ibid., Appendice R.

88. Témoignage de Pierre-Joseph Chevrefils, notaire, 22 décembre 1823, Ibid., Appendice R, Appendice A.

89. Témoignage de James Lambert, 6 décembre 1823, Ibid.

90. Témoignages de Joseph Trépanier, François Ayotte, Pierre Trépanier, 6 décembre 1823, Ibid. 
partenaient pas. Un certain Joseph Deguire, connu aussi sous les noms de Deguire dit Desrosiers ${ }^{91}$ et de Louis Desrosiers ${ }^{92}$, s'était arrogé, vers 1800 , le titre de seigneur de la rivière David et avait prétendu que tout le terrain arrosé par la rivière de ce nom, qui n'avait pas été vendu par ses aỉeux, devait lui appartenir; alors que la seigneurie de ses ancêtres avait été vendue, trente années auparavant, à un nommé Eckart. Son établissement, désigné sous le nom de ruisseau des Chênes, partait des seigneuries Deguire et Bourgmarie de l'est, et s'étendait à travers le Township d'Upton et même un peu dans celui de Grantham ${ }^{93}$. Il avait offert ses terres à un taux relativement faible, et avait réussi de la sorte à s'attacher quelques censitaires. Les gens des environs, voyant qu'il n'était pas inquiété par les autorités, avaient fini par croire à l'authenticité de ses titres". Et pour tout dire, il jouait bien son rôle: "Il y a là des chemins ouverts par le Grand-Voyer du district de Montréal et des Inspecteurs et SousVoyers y sont établis ${ }^{95}$." Si bien qu'en 1823 , le soi-disant seigneur de la rivière David comptait de nombreux censitaires: soixante à quatre-vingts, au dire d'un témoin ${ }^{96}$, une centaine selon d'autres ${ }^{97}$, "tous des Canadiens tant du Nord du fleuve Saint-Laurent que du Sud SB." $^{98}$

D'une part, des obstacles naturels; d'autre part, la spéculation. Les quelques terres propres à la culture et faciles d'accès encore disponibles dans le Bas-Canada seigneurial, une volonté humaine obstinée les soustrayait délibérément aux besoins des nouveaux colons.

Frère MARCEL-JosePh, S.G., M.A.

$$
\text { (a suivre) }
$$

91. Témoignage de Pierre-Basile Pelissier, Ibid.

92. Témoignage de Pierre-Joseph Chevrefils, notaire, 22 décembre 1823, Ibid.

93. Témoignage d'Olivier Arcand, arpenteur, Ibid.

94. Témoignage de Joseph Badeaux, 8 décembre 1823 et d'Olivier Arcand, Ibid.

95. Témoignage de Pierre-Joseph Chevrefils, notaire, 22 décembre 1823, Ibid.

96. Témoignage d'Olivier Arcand, arpenteur, Ibid.

97. Témoignages de Joseph Badeaux, 8 décembre 182łet de Pierre-Joseph Chevrefils, notaire, 22 décembre 1823, Ibid.

98. Témoignage de Pierre-Joseph Chevrefils, 22 décembre 1823, Ibid. 